$57 \mid 2016$

Innovations pédagogiques dans l'enseignement des langues étrangères : perspective historique (XVle-XXIe siècles)

\title{
Apprendre à prononcer le français en Espagne après Galmace (1745) : continuité et nouvelles tendances pédagogiques
}

\section{Marc Viémon}

\section{(2) OpenEdition Journals}

Electronic version

URL: https://journals.openedition.org/dhfles/4415

DOI: $10.4000 /$ dhfles.4415

ISSN: 2221-4038

\section{Publisher}

Société Internationale pour l'Histoire du Français Langue Étrangère ou Seconde

\section{Printed version}

Date of publication: 1 December 2016

Number of pages: 137-155

ISSN: 0992-7654

Electronic reference

Marc Viémon, "Apprendre à prononcer le français en Espagne après Galmace (1745) : continuité et nouvelles tendances pédagogiques", Documents pour l'histoire du français langue étrangère ou seconde [Online], 57 | 2016, Online since 01 February 2018, connection on 28 March 2023. URL: http:// journals.openedition.org/dhfles/4415; DOl: https://doi.org/10.4000/dhfles.4415

This text was automatically generated on 28 March 2023.

All rights reserved 


\title{
Apprendre à prononcer le français en Espagne après Galmace (1745) : continuité et nouvelles tendances pédagogiques
}

\author{
Marc Viémon
}

\section{Situation avant le milieu du XVIII ${ }^{e}$ siècle}

1 Signalons tout d'abord que les ouvrages grâce auxquels les Espagnols pouvaient apprendre la prononciation du français sont assez rares avant la fin du XVII siècle, date à laquelle apparaissent les œuvres de Billet $(1672,1673)$ et de Jaron $(1688)$ : à partir de ce moment-là, les publications de grammaires et autres ouvrages de langue française adressés aux Espagnols ne cesseront plus et augmenteront même progressivement leur nombre.

2 Mais ce point de départ ne représente pas un changement majeur dans la pédagogie de l'enseignement/apprentissage de la prononciation du français. Pour cela, il faudra attendre la publication d'un ouvrage relativement court (80 pages environ) portant uniquement sur la prononciation. Nous voulons parler des Adiciones a la Gramatica Francesa, que compuso el R. P.Nuñez, para el uso de los Cavalleros del Seminario de Nobles, con que brevemente se puede leer, entender, y hablar perfectamente el Idioma Francès, sin auxilio de Maestro d'Antoine Galmace, publiées en 1745 à Madrid et que nous aborderons juste après avoir présenté la situation générale avant le milieu du XVIII ${ }^{\mathrm{e}}$ siècle.

Que ce soit au XVI ${ }^{e}$, au XVII ${ }^{e}$ ou dans la première moitié du XVIII siècle, la section de prononciation dans les grammaires et autres ouvrages «mineurs" consistait en une liste de règles, mélangeant souvent indications de prononciation proprement dites et règles de lecture, et suivant un ordre alphabétique, selon une logique graphophonétique - ou grammatophonique (Besse 2013) -; l'ordre purement 
alphabétique était parfois divisé en deux ou trois sections: voyelles, consonnes et diphtongues.

4 Mais ce qu'il est important de signaler ici, c'est que pratiquement aucun exercice, liste, résumé, tableau récapitulatif ou conseil pédagogique quelconque n'accompagnaient ces indications de prononciation. Tout au plus, les auteurs ont utilisé, en proportion plus ou moins grande, la prononciation figurée pour faciliter l'apprentissage de la prononciation ou lecture/prononciation. Et c'est précisément l'utilisation de la prononciation figurée de façon systématique chez Galmace qui marquera le début d'un changement dans la manière d'enseigner la prononciation du français aux Espagnols ${ }^{1}$.

\section{Avec quoi peut-on apprendre ? L'apparition des exercices d'appui}

\section{1. Les textes}

5 En fait, les premières innovations entrent en consonance avec la volonté de l'auteur, Antoine Galmace, de fournir aux lecteurs un ouvrage d'autoapprentissage : sur la page de titre des Adiciones nous pouvons lire la mention « sin auxilio de maestro ».

6 Ainsi, non seulement l'auteur transcrit tous les exemples qu'il propose dans ses indications de prononciation, mais il inclut également des textes entièrement transcrits, ce qui représente une double nouveauté.

7 Le premier exercice est un texte à caractère historique ${ }^{2}$ d'une quinzaine de pages $^{2}$ (41-67), introduit par une courte introduction intitulée «Demonstracion practica de todas las reglas antecedentes » où il déclare que la lecture du texte est utile pour consolider l'apprentissage des règles et des explications, mais aussi pour la conversation (ce dont nous doutons fortement au vu de la nature du texte ${ }^{3}$ ), la traduction et, surtout, pour exercer les organes articulatoires à proférer des sons et suites de sons inhabituels.

8 Le fait d'insister sur la difficulté articulatoire est d'une grande importance pour nous. Galmace (1745: 41) lui-même est parfaitement conscient de la difficulté musculaire (" qui n'est pas la moindre des circonstances ») inhérente à l'apprentissage de toute prononciation étrangère et pratiquement jamais abordée par les auteurs de grammaire de FLE de cette époque. Seul Chantreau y fera également allusion de façon explicite, nous le verrons.

9 Au sein des Adiciones notre auteur inclut un deuxième texte plus propre à améliorer la compétence communicative orale. En effet, il s'agit de six dialogues représentant des scènes de la vie quotidienne, où Galmace lui-même précise qu'il a utilisé « les mots les plus courants $»^{5}$ (ibid.: 68), preuve de sa conscience pédagogique. Nous transcrivons pour le lecteur les premières répliques du premier dialogue qui reproduit une scène de rencontre, permettant ainsi d'informer l'usager de l'ouvrage des formules de politesse adaptées pour ce genre de situation :

P. Bon jour, Monsieur, comme vous portez vous?

R. Fort bien, pour vous servir.

P. J'en ai bien de la joye.

R. Je vous suis fort obligé.

P. Comme se porte, M. vôtre frere?

R. Il se porte également bien, Dieû merci (idem. : 68-69). 
Dans son ouvrage suivant, une grammaire intitulée Llave nueva, y universal, para aprender con brevedad, y perfeccion la lengua Francesa et publiée en 1748 à Madrid, l'auteur continuera à figurer tous les exemples et inclura même un nouveau texte qu'il affuble du titre « Diálogo español, y francés ». En réalité, le « dialogue » en question n'en est pas vraiment un : le narrateur, qui met le récit en place, introduit deux personnages, un Espagnol et un Français qui se retrouvent en Espagne dans la même auberge puis font route ensemble jusqu'à Saragosse. Chaque voyageur prend la parole à tour de rôle mais leurs répliques sont si longues que ce sont plutôt à des échanges de monologues que l'on assiste. Les sujets de conversation portent surtout sur les clichés concernant trois pays, leurs coutumes et leurs habitants : l'Angleterre, l'Espagne et la France.

11 Contrairement au dialogue transcrit précédemment, ce texte ne reproduit pas les scènes de la vie quotidienne et nous semble donc moins utile que les dialogues de 1745 pour améliorer le niveau de conversation des usagers, même si la notation "phonétique» omniprésente sert toujours de guide pour l'exercice de la lecture/ prononciation.

12 Exactement à la même époque, plus précisément en 1747, Jean-Henri Le Gallois de Grimarest, militaire français de profession, fait publier à Pampelune une grammaire intitulée Nueva gramatica francesa au sein de laquelle il copie vraisemblablement le système de Galmace ${ }^{6}$, transcrivant, sinon l'intégralité, du moins une grande partie des exemples de son ouvrage (jusqu'à la page 187 exactement). Par ailleurs, cet auteur inclut également des textes à la fin de sa grammaire : 20 historietas, de très courts récits censés faire rire ${ }^{7}$.

Il ne transcrit que la première d'entre elles, prétextant que c'était suffisant pour le lecteur pour pratiquer la prononciation $(1747: 632,638)$ et ne s'attarde pas, comme son rival Galmace, sur les explications pédagogiques qui justifient l'inclusion de tels textes, invoquant simplement « le divertissement du lecteur studieux $»^{8}($ ibid. : 632).

Quoi qu'il en soit, le changement pédagogique dans la tradition d'enseignement/ apprentissage de la prononciation du français aux Espagnols commence avec ces deux auteurs, juste avant d'atteindre le milieu du XVIII siècle. D'autres, par la suite, utiliseront également des textes dans leurs ouvrages.

C'est le cas de Soldevila (1767), par exemple, qui inclut à la fin de ses Rudimentos breves y claros para aprender a leer, y escribir en francés sin socorro de maestro une série de prières (30-39) et justifie leur présence en ces termes: "pour que tu parviennes à te familiariser avec cette langue en peu d'efforts, comme tu fais tes prières le matin, et la nuit, je les inclus ici en français de la manière la plus brève possible $»^{9}(1767: 29)$. Soldevila ne précise pas que c'est pour s'entraîner à lire, cependant comme son ouvrage n'est pas une grammaire, mais une cartilla de prononciation, il faut supposer que c'est surtout à ce dessein qu'il a choisi d'introduire ces textes religieux.

16 Au sein de la Cartilla Francesa ó Combinaciones generales para la lectura, González Cañaveras introduit aussi ce type de textes en version bilingue (1781: 38-59), mais sans aucune indication. Sans doute ceux-ci avaient à être lus, certainement sous l'oreille attentive d'un professeur.

En fait, à part les précurseurs Galmace et Grimarest, l'auteur qui mérite réellement un commentaire particulier est Tallés (1773). Dans son ouvrage, le texte de lecture (14-16) conçu comme un exercice est le plus élaboré d'un point de vue de la pratique de la prononciation, encore que le texte en lui-même ne présente certainement pas 
beaucoup d'attraits pour un jeune public: il parle des profits de la conversation, de l'universalité de la langue française et de l'importance de maîtriser les synonymes afin de parler avec justesse. C'est cette maitrise qui, selon l'auteur, «distingue l'homme délicat de l'homme vulgaire » $(1773: 15)$. Mais, mis à part le thème, c'est la première fois que l'on a affaire à un exercice réfléchi et présenté de la sorte.

Tout d'abord, le texte est précédé d'un titre très clair : "Práctica de las Lecciones de este Papel». Ensuite, le commentaire qui précède l'exercice donne des indications précises sur la réalisation de celui-ci :

Tandis que l'on choisira le Livre pour lire, et traduire, le débutant mettra son temps à profit en lisant et en relisant le discours suivant. Dans le premier paragraphe je sépare les syllabes, et les difficultés sont signalées en italiques. Dans le deuxième je rassemble les mots, et je mets seulement le trait d'union entre la consonne finale, qui doit se rattacher à la voyelle du mot suivant, et finalement je continue le discours de manière ordinaire. $(1773: 13)^{10}$

19 Tallés a créé un texte à trois niveaux qui permet aux lecteurs de pratiquer la coupe syllabique et la liaison, pour ensuite s'exercer à lire correctement sans indications ; dans les deux premiers niveaux, par ailleurs, les difficultés phonétiques sont en italiques. Ce qui attire l'attention ici, c'est cette progression que l'auteur propose, preuve qu'il a réfléchi à son enseignement.

Les textes, plus ou moins adaptés et travaillés, sont donc les premières innovations en tant qu'exercices d'appui inclus dans les ouvrages pour mettre en pratique les règles de lecture et explications de prononciation mises en avant par les auteurs. Mais ne sont pas les seules innovations qui apparaissent dès le milieu du XVIII ${ }^{e}$ siècle. Certains grammairiens incluront également des listes dans le but de faciliter l'apprentissage de la prononciation à leurs usagers.

\section{2. Listes}

Roca y María publie une grammaire à Barcelone en 1750 intitulée Arte Francès, ò Nuevo Methodo Facilissimo, para Leer, Hablar, y Escrivir Francès, conforme à lo mas moderno. Il est le premier à inclure une liste contenant des difficultés phonétiques que nous pouvons classer comme un exercice. Elle s'intitule «Lista, ò leccion cotidiana para descanso del Maestro, y provecho del Discipulo » et se compose des « Diphtongues qui posent le plus de problèmes aux Étrangers $»^{11}(1750: 11)$. En réalité, les lettres/sons en question sont «ou, oi, eu, oe, y la vocal $u »$ (ibid.).

Cette liste est précédée de recommandations. Non seulement Roca recommande de pratiquer ces sons jugés difficiles tous les jours avant de commencer à lire, mais en plus la description du processus est élaborée : il ne faut pas lire toujours la liste de la même façon, mais varier afin d'obtenir une vraie maîtrise et non pas un apprentissage machinal, automatique ${ }^{12}$.

Tallés, dont nous avons évoqué l'œuvre précédemment, fournit lui aussi des listes de mots pour «s'exercer à la prononciation des différents uus de la langue française $»^{13}$ (1773 : 11). Les lettres/sons en question sont $\mathrm{U}$, EU et OU, presque les mêmes en fait que chez Roca y María, ce qui pourrait laisser penser que Tallés s'en est inspiré. Après trois premières listes où les lettres sont séparées, la quatrième regroupe les différentes possibilités dans le but, certainement, de faire travailler l'élève, encore une fois, de 
façon moins machinale: "Affectueusement, affectueux, aujourd'hui, bienheureux, chou-fleur, couleur, coureur, curieux, etc. » (ibid. : 12).

Dans tous les cas, ces listes de mots étaient à répéter en présence du maître, puisqu'au sujet de ces sons, l'auteur déclare que " pour cette leçon les explications sont inutiles, ce serait apprendre à faire des gestes. La voix du Maître est indispensable, ainsi qu'une pratique assidue $»^{14}$ (ibid. : 11).

Nous trouvons un autre exemple de l'usage de listes conçues comme un exercice chez Pelleport, un auteur de fin de siècle. Il propose au lecteur deux courtes listes de syllabes mettant chacune en regard $\mathrm{B}$ face à $\mathrm{V}$ et $\mathrm{CH}$ face à $\mathrm{J}$, précédées du commentaire suivant: « Pour que l'on comprenne la différence qu'il existe en français entre $B$ et $V$, et entre $c h$ et $J$, je dispose les deux lignes suivantes $»^{15}\left(\right.$ f. XVIII $\left.I^{\mathrm{e}}: 3\right)$. Sans aucun doute, la répétition de ces listes exigeait également la correction d'un maître.

Mais le travail sur les listes est poussé à son plus haut degré chez Chantreau, au sein de l'Arte de hablar bien francés, ó Gramatica completa publié à Madrid en 1781. Tallés parlait bien d'« exercitar " pour sa liste et de "práctica » pour son texte, et Galmace de "Demonstracion practica ", ce qui prouve, chez ces auteurs, la volonté de marier la théorie à la pratique ; Chantreau, lui, intitule carrément ses listes « Exercicio diario que debe hacer el discipulo para perfeccionarse en la pronunciacion del francés » (1781: 36-42). Dès le titre, il explique que l'élève doit répéter les listes qu'il propose tous les jours, de même que Roca y María, rappelons-le.

De plus, l'exercice de répétition de Chantreau est le plus complet de notre corpus : il met l'accent sur certaines difficultés comme Roca y María ou Tallés, mais pas seulement. En effet, les deux premières listes correspondent respectivement à toutes les voyelles et à toutes les consonnes. Puis il fournit quatre listes supplémentaires correspondant aux oppositions entre $/ \mathrm{b} /$ et $/ \mathrm{v} /, \mathrm{z} /$ et $/ \mathrm{s} /, \mathrm{y} /$ et $/ \mathrm{u} /{ }^{16}$ et entre longues et brèves, en ciblant donc les erreurs les plus récurrentes pour lui.

Par ailleurs, chaque opposition est illustrée par un grand nombre de paires minimales (ante litteram), ce qui démontre que Chantreau est non seulement un pédagogue, mais aussi un fin linguiste, ou du moins un grammairien au fait des ouvrages contemporains. Il faut, en outre, souligner que la raison qu'il donne pour justifier la répétition quotidienne de ces listes est très rare. Nous l'avons évoquée à propos du premier texte de Galmace. En effet, Chantreau, en tête des quatre listes, déclare : «Pour acquérir une bonne prononciation, il est nécessaire de réaliser un travail physique, pour obliger la langue à articuler sans effort les sons les plus éloignés de la langue maternelle ${ }^{17}$ (ibid. : 36). Les autres auteurs de l'époque - Galmace excepté - ne mentionnent jamais le fait que la répétition est bonne, non pas seulement pour la mémoire articulatoire, mais aussi et simplement en ce qui concerne la musculature à mettre en jeu pour prononcer des sons ou des combinaisons sonores inhabituels.

Jusqu'ici nous avons abordé les nouveautés en matière d'exercices accompagnant les explications de prononciation. Mais les innovations dans les ouvrages de la seconde moitié du XVIII siècle ne s'arrêtent pas là. En effet, dans ces ouvrages, une conscience pédagogique explicite fait son apparition et se trouve reflétée par des conseils d'utilisation des œuvres, une hiérarchisation des contenus, des synthèses et des tableaux récapitulatifs. C'est ce que nous allons aborder maintenant. 


\section{Comment faut-il apprendre ? L'apparition d'une conscience pédagogique explicite}

31 Roca y María, de nouveau, est le premier à vouloir expliquer aux usagers de sa grammaire la façon dont ils doivent apprendre «la Prononciation, et Orthographe Française rapidement $»^{18}$ (1750 : s. n.). C'est au sein de l'« Aviso al lector » qu'il énumère sa méthode en cinq étapes :

On étudiera tout d'abord les Règles de la Prononciation, suivies de celles de l'Orthographe, qu'il faudra étudier soigneusement, et puisqu'elles constituent la base du discours correct oral et écrit, on tâchera de ne pas les oublier [...].

2. On prendra un Livre Français (moderne), et au cours de sa lecture, on s'efforcera de noter les fautes d'Orthographe, s'il y en a dans ses mots.

3. Après avoir assimilé les Règles, on poursuivra la lecture, avec une attention telle que l'on recueille le fruit des observations que l'on y fera.

4. On étudiera les Conjugaisons des Verbes [...].

5. Dans le cas où, en lisant, on trouverait deux, ou trois termes qui, bien qu'orthographiés différemment, se prononcent de la même manière, on ira voir leurs sens au Cap. XV. fol. 221. même s'il y manquera sans doute beaucoup d'homonymes, malgré toute l'attention que j'ai portée à les chercher. (1750 : s. n.) ${ }^{19}$

Nous le voyons, chez Roca y María l'orthographe occupe une place prépondérante. Il attache, par exemple, beaucoup d'importance aux homonymes. Cependant, en ce qui concerne la façon de travailler la prononciation, il ne fournit pas à l'usager de véritable méthode: après avoir recommandé d'apprendre tout d'abord les règles de la prononciation et ensuite celles de l'orthographe, il ne mentionne, au sein du point numéro trois, que l'exercice de la lecture, sans préciser la nature de ce qui doit être lu, la fréquence avec laquelle il faut lire, ni réellement comment il faut lire.

Rousseau, en revanche, dans ses Rudimentos de la Lengua Francesa publiés en 1754 à Valladolid, prétend que la lecture doit se réaliser à petites doses de façon quotidienne : «Les premiers jours, où le Disciple manipule le livre, il faudra lui faire lire très peu de lignes, en le chargeant d'appliquer les Règles, dont il connaît la théorie $»^{20}(1754: 64)$. Cela mis à part, sa méthode n'est pas très originale : l'élève lit et le maître le reprend, " en lui imprimant l'accent, en corrigeant ses défauts " ${ }^{21}$ (ibid.). Pourtant, à la fin des explications sur chacune des lettres de l'alphabet, il prend la peine de préciser que la progression est essentielle dans le processus d'apprentissage et que le maitre doit adapter son enseignement à chaque élève :

Il est important d'avoir à l'esprit toutes ces différences, qui sont simples en théorie, mais très difficiles en pratique les premiers jours, ainsi l'abécédaire servira de travail, pour plus ou moins de leçons, en proportion avec la capacité, la disposition, ou les occupations du Disciple, en veillant à ne jamais passer à la deuxième leçon, sans qu'il ne possède bien la première, ce qui serait du temps perdu, sans une maîtrise solide de la base ; on continuera tous les jours avec la même méthode, et le Maître, pouvant mesurer les talents de son Disciple, devra distribuer les leçons selon ses forces. (Ibid. : 11) $)^{22}$

Rousseau, par ailleurs, est l'un des premiers à s'adresser aussi bien aux élèves qu'aux maîtres qui utiliseraient son ouvrage. C'est aussi le cas de Tallés qui proscrit l'exercice de la lecture pour un élève qui ne connaîtrait pas préalablement les règles :

...remettre un Livre Français, dès le premier jour au disciple qui débute, pour qu'il y apprenne les premiers rudiments de la prononciation, est une méthode désastreuse. Comme celui qui essaie d'apprendre une autre langue sait déjà lire dans la sienne, il trouve qu'épeler est propre aux enfants, et il est clair qu'il veut lire de manière 
suivie. Le Maître se fatigue inutilement à le reprendre, et le Disciple s'agace de ces interruptions continuelles. Une fois surmontées les difficultés de ce Livret, il ne sera pas nécessaire de le couper sans arrêt lorsqu'il commencera à lire ${ }^{23}$. (1773:3) correspond une méthode d'apprentissage particulière. Sans doute, cette observation est moderne et dénote une conscience pédagogique propre, pouvant s'apparenter à celles de Rousseau et de Chantreau. Mais elle n'est pas très développée chez notre auteur. En effet, elle se résume simplement à adapter l'information selon que l'élève est «meilleur » ou «moins bon » et suivant une prémisse simple: meilleur est l'élève et plus il peut assimiler de règles. Ainsi, il précise dans l'« Aviso » que les bons élèves devront étudier les contenus développés (" en Leçons, pour les étudier par cœur " ${ }^{27}$ ) ; l'option deux s'adresse à ceux qui n'ont pas le courage de tout étudier: la prononciation quotidienne de l'alphabet et le travail sur un résumé de 19 lignes comportant des exemples suffit. Quant à l'option trois, pour les plus faibles, c'est une courte liste de lettres et de combinaisons graphiques qui n'inclut même pas d'exemples, et certainement peu utile.

41 Finalement, le dernier auteur à manifester une conscience pédagogique de façon explicite est Boyer, qui publie sa grammaire, le Método facilísimo para leer y hablar 
francés, en 1799 à Barcelone. Il recommande également d'apprendre les règles par cœur avant de commencer à lire en articulant chaque mot mais précise que l'élève doit lire un livre bilingue pour se reposer quelque peu de cet exercice que l'auteur qualifie luimême d'« aride et ennuyant $»^{28}(1799: 5)$, mais qui, selon lui, porterait ses fruits. Par ailleurs, il recommande lui-même certains livres qui lui semblent appropriés: «le Télémaque, l'abrégé de l'histoire d'Espagne du Père Duschene ou le Catéchisme de Fleuri $»^{29}(i b i d$.$) , courants à l'époque { }^{30}$.

\section{Conclusions}

En guise de conclusion, nous aimerions mettre deux idées complémentaires en relief. Tout d'abord, si Chantreau a bien démontré dans sa grammaire qu'il était un grammairien doté d'une conscience pédagogique poussée, son témoignage n'est pas isolé, loin de là. En effet, nous avons vu qu'un certain nombre d'auteurs de la deuxième moitié du XVIII siècle ont pris la peine de réfléchir aux méthodes que devaient suivre les apprenants dans leur apprentissage et aux outils qu'ils pouvaient utiliser. Bien avant Chantreau, c'est vers le milieu du XVIII ${ }^{e}$ siècle que commence à se développer l'explicitation d'une certaine conscience pédagogique. Galmace en serait l'auteur " charnière ".

La deuxième idée que nous aimerions mettre en valeur est la suivante : l'apparition, dans les grammaires et autres ouvrages de prononciation, d'exercices tels que des textes ou des listes visant à améliorer la prononciation des élèves est loin d'être évidente à l'époque où nous situons notre étude. Dans la tradition italienne des ouvrages de FLE, par exemple, Minerva (1997: 54) affirme que "les exercices de prononciation sont totalement absents dans les manuels jusqu'en 1805 ». Nous savons maintenant que c'est loin d'être le cas en Espagne.

Il y aurait bien d'autres choses à dire sur l'arrivée de nouvelles tendances pour l'apprentissage de la lecture/prononciation dans la deuxième moitié du XVIII siècle, comme l'apparition d'une mode syllabique, par exemple, mais nous laisserons ces réflexions pour un prochain travail.

\section{BIBLIOGRAPHY}

\section{Sources primaires}

BILLET, Pierre-Paul (1672). Arte para aprender facilmente, y en poco tiempo a leer, escrivir, y hablar la Lengua Francesa. Lyon : Pierre Bourgeat.

Billet, Pierre-Paul (1673). Gramatica Francesa, dividida en dos partes. Sarragosse : s. n.

BOYER, Maurice-Ignace-François (1799). Método facilísimo para leer y hablar francés. Barcelone :

Antonio Sastres.

BUFFIER, Claude (1711) [1709]. Grammaire françoise sur un plan nouveau. Bruxelles : Jean Léonard. 
CHANTREAU, Pierre-Nicolas (1781). Arte de hablar bien francés, ó Gramatica completa. Madrid : Antonio de Sancha.

JARON, Jean-Pierre (1688). Arte nuevamente compuesto de la Lengua Francesa por la Española. Madrid : Lucas Antonio de Bedmar y Baldivia.

GALMACE, Antoine (1745). Adiciones a la Gramatica Francesa, que compuso el R. P.Nuñez, para el uso de los Cavalleros del Seminario de Nobles, con que brevemente se puede leer, entender, y hablar perfectamente el Idioma Francès, sin auxilio de Maestro. Madrid : s. n.

GALMACE, Antoine (1748). Llave nueva, y universal, para aprender con brevedad, y perfeccion la lengua Francesa, sin auxilio de Maestro, que procede por todas las partes de la oracion, añadiendo frasses para la inteligencia del uso de ellas, adornada de una Recopilación de los Verbos, y Terminos mas necessarios, pertenecientes à diversas Artes, y Facultades; y acaba con un Dialogo muy gustoso, y abundante. Madrid : Gabriel Ramírez.

GONZÁLEZ CAÑAVERAS, Juan Antonio (1781). Cartilla Francesa ó Combinaciones generales para la lectura. Cadix : Luis de Luque y Leyva.

GRIMAREST, Jean-Henri Le Gallois de (1747). Gramatica Francesa con un nuevo Methodo para aprender a pronunciar. Pamplona : Herederos de Martínez.

PELLEPORT, Dionisio de (f. XVIII ${ }^{\mathrm{e}}$ ). Reglas para pronunciar, y leer con inteligencia la lengua francesa.

Madrid : s. n.

ROCA Y MARÍA, Sébastien (1750). Arte Francès, ò Nuevo Methodo Facilissimo, para Leer, Hablar, y Escrivir Francès, conforme à lo mas moderno. Barcelone : Francisco Surià.

RousSEAU, Paul-François (1754). Rudimentos de la Lengua Francesa, ò extracto de preceptos breves, y claros para su prompta inteligencia. Valladolid: Alonso de Riego.

SOLDEVILA, Francisco (1767). Rudimentos breves y claros para aprender a leer, y escribir en francés sin socorro de maestro. Madrid : Antonio Muñoz del Valle.

TALLÉs, Juan Magín (1773). Rudimentos de la pronunciacion Francesa, para el uso de los Caballeros del Real Seminario de Nobles de esta Corte. Madrid : Antonio Mayoral.

\section{Sources secondaires}

BESSE, Henri (2013). « Contribution à l'histoire des pratiques de 'prononciation' fondées sur les écritures ordinaires ». Documents pour l'histoire du français langue étrangère ou seconde, 50, 117-138.

GARCIA BASCUÑANA, Juan Francisco (2005). « Materiales para la enseñanza del francés en España : aproximación a los manuales publicados entre los siglos XVI y XX ». Revista Interuniversitaria de Formación del Profesorado, 53, 129-144.

MinERVA, Nadia, (1997). «Les sons nasaux enseignés aux Italiens aux $17^{\mathrm{e}}$ et $18^{\mathrm{e}}$ siècles ». Documents pour l'histoire du français langue étrangère ou seconde, 19, 51-64.

VIÉMON, Marc (2013). «Le traitement de $e$ dans un système de prononciation figurée au XVIII ${ }^{\mathrm{e}}$ siècle : la Coleccion de las falsas reglas de Galmace ». Çédille, 9. En ligne :

<http://cedille.webs.ull.es/9/29viemon.pdf> (23 août 2016). 


\section{NOTES}

1. García Bascuñana (2005: 136) reconnaissait déjà, de manière générale, le rôle de charnière («obra 'puente'») des manuels de Galmace entre ceux de Núñez de Prado (1728) et de Chantreau (1781), et «l'importance de la signification pédagogique et méthodologique de Galmace » (« la importancia de la significación pedagógica y metodológica de Galmace »).

2. Le texte relate certains faits de la guerre entre l'empire ottoman et la République de Venise, dans laquelle intervient l'empire d'Autriche, jusqu'à la signature du traité de Passarowitz en 1718.

3. Voici la première phrase de ce texte : "L'heûreûx sûccès, que les Tûrcs tirerent de la Guerre qu'ils entreprirent contre la Rûssie par la sollicitation dû Roy de Sûede, les dissensions intestines de la Pologne, l'épuisement de l'Empereûr, étoient autant de raisons qui les engagea à declarer la guerre a la Repûblique de Venise » $(1745: 41)$.

4. « que no es la menor circunstancia ».

5. « las voces mas regulares».

6. Cette imitation donnera lieu à une violente polémique que nous ne pouvons aborder ici. Pour plus d'informations, nous renvoyons à Viémon (2014).

7. Nous reproduisons ici quelques titres de ces histoires pour donner une idée de leur contenu : «L'âne qui fait son maître menteur »; «Le vieux soupirant »; «Le buveur malade »; «Le curé ignorant»; etc.

8. « el recreo del estudioso lector».

9. «para que con poco trabajo logres familiarizarte con este idioma, supuesto que haces la pregaria de mañana, y noche, la pongo aqui en Francès con el mas breve estilo ».

10. «Mientras se determina de que Libro se echará mano para leer, y traducir, emplearà muy bien su tiempo el principiante que leyere una, y muchas veces el siguiente discurso. En el primer parrafo separo las sylabas, y las dificultades ván de letra bastardilla. En el segundo ùno yá las palabras, y solo pongo guión entre la consonante final, que debe unirse con la vocal de la palabra siguiente, y luego prosigo el discurso de modo regular ».

11. «Diphtongos, en los quales el Estangero halla mas dificultad».

12. «La siguiente Lista se leerá todos los dias antes de empezar à leer en el Libro, leyendo primero todas las quatro Colunas, cada una de por sì ; y despues variando con seguir el renglon de arriba abaxo; esto es, tomando el primero de la primera Coluna, despues el primero de la segunda, \&c. y de de esta suerte bolviendo à leer todos los terminos contenidos en ellas ; lo que será de un grande provecho, para la pronunciacion, ò accento, yugo indispensable de todas las Lenguas » $(1750: 12)$.

13. « exercitar la pronunciacion de las diferentes uus de la Lengua Francesa ».

14. « para esta leccion no sirven explicaciones, sería enseñar á hacer gestos. Es indispensable la voz del Maestro, y mucha practica».

15. «Para que se comprehenda la diferencia que hay en frances entre B y V, y entre ch y J, pongo las dos líneas siguientes ».

16. Chantreau, bien entendu, ne connaît pas le concept moderne de phonème. Nous utilisons ici ces notations qui nous semblent plus adéquates pour parler d'oppositions.

17. «Para facilitarse una buena pronunciacion, es menester hacer de ella un estudio fisico, para obligar la lengua à soltarse sin trabajo en la pronunciacion de los sonidos mas estraños à la lengua nativa ».

18. « la Pronunciacion, y Orthographia Francesa con brevedad».

19. «Se estudiarà Primeramente las Reglas de la Pronunciacion, despues las de la Orthographia, haziendo reflexion sobre ellas, que por ser la basis del bien hablar, y escrivir, se pondrà cuydado en que no se olviden [...]. 
2. Se tomarà un Libro Francès (moderno), y leyendo en él, se irà con la atención de notar las faltas de Orthographia, si alguna hay en sus terminos.

3. Despues de sabìdas las Reglas, se continuarà siempre la lectura, que ha de ser con tal atencion, que las observaciones, que se haràn en ella, produzgan el fruto de su trabajo.

4. Se estudiaràn las Conjugaciones de los Verbos [...].

5. Quando leyendo se hallaràn dos, ò tres terminos, que aunque diversamente escritos, se pronuncian de la misma suerte el uno, que el otro, se irà à ver sus significados en el Cap. XV. fol. 221. aunque no dudo, que muchos Equivocos faltaràn en èl, no obstante el cuydado, que he tenido en buscarlos ».

20. «Los primeros dias, que toma el Discipulo el libro en la mano, se le haran leer muy pocos renglones, haciendole cargo de la aplicacion de las Reglas, que sabe por thèorica ».

21. « infudiendole el acento, corrigiendole sus defectos».

22. « Es importante el tener presentes todas estas diferencias, que son faciles en la thèorica, pero muy dificultosas en la practica los primeros dias, assi el abècedario servirà de tarea, para mas, ò menos lecciones, à proporcion, de la capacidad, inclinacion, ò ocupaciones del Discipulo, nunca passando à segunda leccion, sin que possea bien la primera, lo contrario serìa tiempo perdido, el no radicarle bien en los fundamentos ; y se seguirà todos los dias el mismo methodo, siendo el Maestro, quien como juez de los talentos de su Discipulo, debe proporcionarse à sus fuerzas en la distribucion de sus lecciones ».

23. « el entregar un Libro Francés, desde el primer dia al discipulo que empieza, para que en él aprenda los primeros rudimentos de la pronunciacion, es un malisimo método. Como yà sabe leer en su lengua el que intenta aprender otra, le parece cosa de niños el deletrear, y quiere desde luego leer corriente. El Maestro se cansa inutilmente en reprehender, y el Discipulo se enfada de verse tan amenudo interrumpido. Vencidas las dificultades de la pronunciacion en esta Cartilla, no habrá que detenerle à cada paso quando se ponga á leer ».

24. En ce qui concerne l'adaptation des contenus en fonction du niveau des élèves, il se peut que Chantreau se soit inspiré de Buffier. En effet, ce dernier déclarait au sein de la préface : «J'ai inséré en cette seconde partie des réfléxions qui ne sont pas si nécessaires, pour l'usage ordinaire, je les ai fait désigner par une marque * particuliere afin qu'on puisse les omettre, sans perdre la suite générale des regles. Les Commençans pourront passer ces réfléxions la premiere fois qu'ils liront cette Grammaire pratique ; afin de ne point rebuter par un trop grand nombre d'observations, \& de se familiariser davantage avec les régles les plus génerales. On fera bien même de ne lire que tres-peu de regles à la fois pour prévenir le dégout » (1711: s. n.).

25. «Resumen de las reglas de la pronunciacion que he reducido en una sola plana [...]. Para que de un lado el principiante tenga su libro, y de otro, todas las reglas magistrales de que se necesita para leerlo ».

26. «porque el hablar enteramente depende de la práctica ; y por hábil que sea el Maestro, nada o poco puede sin ella ».

27. « en Lecciones, para estudiarlas de memoria ».

28. « arido y enfadoso".

29. « el Telemaco, el compendio de la historia de España del Padre Duchesne ó el Catescismo de Fleuri ».

30. Rousseau (1754: 63) recommandait également des livres (« las obras del Señor Bussi Rabutin, el Thèlèmaco, los Tratados del Padre Regnault, el de la Opinion, Cartas Familiares, las Historias de el Señor Rollin, \&c. ») mais c'était pour l'exercice de la traduction. 


\section{ABSTRACTS}

In French as a Foreign Language texts published in the second half of the 18th century and aimed at Spanish readers, specifically after the publication, in 1745, of Adiciones by Antoine Galmace, there appear many pedagogical innovations in pronunciation sections. These innovations manifest themselves by the inclusion of reading and pronuciation exercises, tips from the authors on how to use the works, adaptations of the content to each learner's level, summary tables and syllabaries; the latter almost certainly an import from France. The fact that the publication of pronunciation cartillas is concentrated in the second half of the 18th century is not a coincidence either. The aim of this essay is to demonstrate a clear shift from the almost complete absence, in the 17th century and in the first half of the 18th century, of pedagogical processes and techniques intended to simplify teaching French pronunication in French as a Foreign Language books aimed at Spanish learners, to the proliferation of these same innovative processes and techniques in the second half of the 18th century.

Au sein des ouvrages de FLE publiés dans la deuxième moitié du XVIII ${ }^{\mathrm{e}}$ siècle à l'adresse des Espagnols - et plus exactement à partir de 1745, date de publication des Adiciones d'Antoine Galmace -, de nombreuses innovations pédagogiques font leur apparition dans la section de prononciation. Ces innovations se matérialisent par l'inclusion d'exercices de lecture/ prononciation, de conseils d'utilisation des œuvres de la part des auteurs, d'adaptation des contenus au niveau de chaque usager, de tableaux récapitulatifs et autres, comme les syllabaires sans doute importés de France. Le fait que la publication de Cartillas de prononciation se concentre dans la deuxième moitié du XVIII ${ }^{\mathrm{e}}$ siècle n'est pas non plus un hasard. Le but de cet article est de mettre en évidence cette évolution claire entre l'absence plus ou moins généralisée, au XVII ${ }^{\mathrm{e}}$ et dans la première moitié $\mathrm{du} \mathrm{XVIII}^{\mathrm{e}}$ siècle, de procédés et techniques pédagogiques visant à faciliter l'enseignement de la prononciation française dans les manuels de FLE adressés aux Espagnols, et l'essor de ces mêmes procédés et techniques, innovants, dans la deuxième moitié du XVIII ${ }^{\mathrm{e}}$ siècle.

\section{INDEX}

Keywords: French pronunciation, history of language learning and teaching (HoLLT), Spain, 18th century

Mots-clés: prononciation du français, histoire de la didactique des langues, Espagne, XVIIIe siècle

\section{AUTHOR}

\section{MARC VIÉMON}

Université de Séville

mviemon@us.es 